\title{
Análisis del mercado Spot con representación estocástica para generación eólica y fotovoltaica
}

\section{Spot market analysis with stochastic representation for wind and photovoltaic generation}

\author{
Jorge Alexander Alarcón-Villamil ${ }^{1}$, Sergio Raúl Rivera-Rodríguez ${ }^{2}$, Francisco Santamaría-Piedrahita $^{3}$ \\ ${ }^{1}$ Grupo de Compatibilidad e Interferencia Electromagnética-GCEM, Ingeniería Eléctrica, Universidad Distrital Francisco José de \\ Caldas, Colombia. \\ Orcid: 0000-0002-8718-2542. Email: jaalarconv@udistrital.edu.co \\ ${ }^{2}$ Grupo de investigación en compatibilidad electromagnética-EMC, Ingeniería Eléctrica y Electrónica, Universidad Nacional de \\ Colombia, Colombia. \\ Orcid: 0000-0002-2995-1147. Email: srriverar@unal.edu.co \\ ${ }^{3}$ Grupo de Compatibilidad e Interferencia Electromagnética-GCEM, Ingeniería Eléctrica, Universidad Distrital Francisco José de \\ Caldas, Colombia. Orcid: 0000-0002-0391-4508. Email: fsantamaríap@udistrital.edu.co
}

RECIBIDO: Febrero 17, 2017. ACEPTADO: Ju1io 24, 2017. VeRSIÓN FINAL: Octubre 20, 2017.

\section{RESUMEN}

Este artículo analiza el impacto que tienen las plantas de generación fotovoltaica y eólica en el precio del mercado Spot cuando se tiene un modelo de despacho de energía no liberalizado y se usa una estrategia de despacho que da beneficios a las plantas renovables, permitiéndoles despachar con un precio de oferta igual a cero. La técnica de Replicator Dynamics usa la utilidad marginal de las plantas para optimizar el despacho, llegando al óptimo cuando las plantas alcanzan la misma utilidad marginal. El estudio también incluye la representación estocástica de la velocidad del viento y la radiación solar para analizar las variaciones en la utilidad marginal cuando se tiene generación eólica, cuando se tienen generación fotovoltaica y cuando se incluyen los dos sistemas de generación de forma simultánea. Los resultados indican que la estrategia de despacho genera variaciones hasta de un $21.9 \%$ en la utilidad marginal, afectando a las plantas en firme con reducciones de hasta un $58.6 \%$ en la potencia despachada cuando se tiene únicamente Generación Fotovoltaica e incluso haciendo que disminuya a cero cuando se tiene generación eólica.

PALABRAS CLAVE: Despacho de potencia; mercado spot; análisis estocástico; generación fotovoltaica; generación eólica.

\footnotetext{
ABSTRACT

This paper analyzes the impact of photovoltaic and wind power plants on the price of Spot market, when a nonliberalized energy market and a profit-based dispatch strategy for renewable plants are used, that allow them dispatching the power at an offer price equal to zero. The Replicator Dynamics technique uses marginal utility of the power plants to optimize the dispatch, reaching the optimal when marginal utility of the plants become equal. The study also includes stochastic representation for wind velocity and solar radiation to analyze the variations in the marginal utility for three cases: including wind power plants, including photovoltaic power plants, and including both ISSN Impreso: 1657 - 4583, En Línea: 2145 - 8456

Este artículo puede compartirse bajo la licencia CC BY-ND 4.0 y se referencia usando el siguiente formato: J. Alarcón, S. Rivera, F. Santamaría, "Análisis del mercado Spot con representación estocástica para generación eólica y fotovoltaica," Rev. UIS Ing., vol. 17, no. 1, pp. 155-162, 2018. Doi: https://doi.org/10.18273/revuin.v17n1-2018014
} 
wind and photovoltaic power plants simultaneously. Results indicate that the dispatch strategy generates variations of up to $21.9 \%$ in the marginal utility, affecting the thermal plants with reductions of up to $58.6 \%$ in the power delivered when Photovoltaic Generation is included, and even causing this power fall to zero when wind power generation is included.

KEYWORDS: Power dispatch; spot market; stochastic analysis; photovoltaic generation; wind generation.

\section{INTRODUCCIÓN}

El mercado de energía eléctrica se encarga de establecer mediante mecanismos de oferta y demanda, el precio al que se paga cada unidad de potencia despachada hora a hora, durante todo el día, y las plantas de generación asignadas para el despacho con las respectivas cantidades.

Entre los aspectos requeridos para el despacho se tienen en cuenta el precio de oferta o la función de costos de cada una de las plantas, y la disponibilidad de potencia despachable, que para el caso de las plantas de generación firme tiende a mantenerse constante durante todo el día. Este aspecto difiere en las plantas intermitentes porque en estas, la cantidad de potencia generada depende de las condiciones ambientales (radiación solar y velocidad del viento) y además, puede cambiar de manera repentina [1][2].

Uno de los aspectos que impulsa el crecimiento de las energías renovables es que los costos de instalación y operación han disminuido significativamente en los últimos 20 años, y esto ha reducido los costos de generación [3], sin embargo, estos dependen de muchos factores como la ubicación geográfica, el tipo de tecnología utilizada, la disponibilidad del recurso primario (radiación solar y velocidad del viento) [4], la regulación de cada país y la potencia instalada [5]. Esto hace que en algunos casos los costos de generación sean competitivos con tecnologías como plantas térmicas a gas, mientras que en otros casos no se logran estas condiciones.

Con la integración de los sistemas de generación distribuida se ha generado la necesidad de evaluar además del despacho, nuevos aspectos como lo son el tránsito y congestión de las redes [6], la regulación de la frecuencia frente a los cambios de demanda y generación [7], y las maneras de generar reducción en el consumo con estrategias de gestión de demanda para modificar las curvas de demanda diaria en distribución [8]. Estos aspectos se ven influenciados tanto por las plantas de generación en firme como las de generación intermitente.

Debido a la integración de las plantas de generación intermitente como la generación eólica (GEo) y la fotovoltaica $(\mathrm{GFv})$, se han modificado las metodologías de despacho tradicionales para incentivar a los inversionistas privados a participar en este mercado [9] [10]. Una de las estrategias usadas es permitir que las plantas renovables participen con un precio de oferta igual a cero, para garantizarles el despacho de la potencia disponible en todo momento, evitando que compitan libremente en el mercado con las otras tecnologías de generación [11] [12]. Dicha estrategia es evaluada en este artículo mediante representación estocástica de la radiación solar y velocidad del viento, para finalmente ser optimizada usando una técnica basada en la teoría de juegos conocida como "Replicator Dynamics".

La importancia de este tipo de estudios radica en la necesidad de conocer y evaluar las metodologías utilizadas para el despacho de energía, identificar los beneficios y problemas que puedan tener, e identificar las metodologías que se pueden implementar en países como Colombia, que están empezando a implementar estas tecnologías de generación. El conocimiento adquirido por países como España y Alemania, que ya han tenido experiencias con errores y aciertos en este aspecto, pueden aportar mucho para nuestro conocimiento a fin de evitar los problemas que estos ya enfrentaron y solucionaron.

Con el análisis estocástico de las variables asociadas a la GEo y GFv se pueden identificar efectos y consecuencias en el despacho económico, como lo son: la reducción en la potencia que despachan las plantas en firme y un incremento en la utilidad marginal cuando se aumenta la potencia entregada por las plantas renovables al sistema.

Este artículo se divide en cinco secciones organizadas como se indica a continuación. En la primera se hace la introducción al tema. La segunda muestra la manera como se hace la representación estocástica del recurso primario para las plantas renovables. La tercera parte presenta el caso de estudio, indicando las características de la microrred de prueba y el tipo de plantas utilizadas. En la sección cuatro se hace el planteamiento del problema, mostrando la representación matemática de la técnica de Replicator Dynamics, las restricciones de las plantas y la red, y la función objetivo. En la sección cinco se presentan los resultados obtenidos para los casos de estudio planteados y en la sección seis se presentan las conclusiones del estudio. 


\section{REPRESENTACIÓN DE LAS PLANTAS RENOVABLES}

Uno de los aspectos más importantes a evaluar en este articulo es, cómo la intermitencia de los sistemas de GEo y GFv impacta el comportamiento del mercado de energía, por lo cual se requiere una adecuada representación de las variables ambientales (radiación solar y velocidad del viento).

\subsection{Representación estocástica de las variables}

Para el caso de estudio se realiza la presentación estocástica de la velocidad del viento y la radiación solar, que se usan como datos de entrada en el cálculo de la potencia eléctrica del GEo y el GFv.

La función Weibull representa la frecuencia de repetición de las velocidades de viento, y permite establecer la probabilidad de ocurrencia de una velocidad dada [13]. Para el caso de estudio los parámetros de la función Weibull son [14]:

Factor de forma: $\mathrm{k}=2$

Factor de escala: $\mathrm{c}=63.74$

La radiación solar puede tener cambios repentinos en su magnitud debido a factores ambientales como nubosidad, que afecta la potencia generada y los precios del mercado. Esta radiación se representa siguiendo una función $\log$ normal [15] con valor medio $\mu=6$ y una desviación estándar $\sigma=0.25$.

\subsection{Potencia del generador eólico (GEo)}

Depende de las características constructivas y los límites de operación de la turbina, así como la velocidad del viento. Para el caso de estudio se toman datos técnicos de una turbina eólica con altura de 100 metros, cuyas características operativas se indican a continuación [15] [16].

Velocidad de arranque: $\mathrm{Vi}=5 \mathrm{~m} / \mathrm{s}$

Velocidad nominal: $\mathrm{Vr}=15 \mathrm{~m} / \mathrm{s}$

Velocidad de parada: $\mathrm{Vo}=45 \mathrm{~m} / \mathrm{s}$

Potencia nominal: $\mathrm{P}_{\text {nom }}=$ varía según el caso

La potencia de salida del generador depende del rango de velocidad en el que opera la turbina, y se calcula como se indica a continuación [17].

$$
\begin{array}{ll}
V i \leq v e l \leq V r, & \text { entonces } P g=a * \text { vel }+b \\
V r<\text { vel }<V o, & \text { entonces } P g=P_{\text {nom }} \\
\text { vel } \leq V i \quad \text { o vel }>V o & \text { entonces } P g=0
\end{array}
$$

donde:

a: coeficiente lineal del aerogenerador

vel: velocidad del viento

b: coeficiente lineal

Pg: potencia del generador eólico

\subsection{Potencia del generador Fotovoltaico (GFv)}

Depende de la capacidad máxima instalada en el arreglo fotovoltaico, la radiación solar que llega al panel y la eficiencia del sistema [15] [16].

Rango de salida del generador $\mathrm{Psr}=100 \mathrm{~W} / \mathrm{m}^{2}$

Valor referencia de la irradiancia $\mathrm{Rc}=750 \mathrm{~W} / \mathrm{m}^{2}$

La potencia entregada por el GFv se determina teniendo en cuenta la irradiancia incidente sobre el panel.

$$
\begin{array}{ll}
0 \frac{W}{m^{2}} \leq R_{s o l} \leq R c \frac{W}{m^{2}} & P_{F V}=P s r * \frac{\left(R_{s o l}\right)^{2}}{G_{s t d} * R c} \\
R_{s o l}>R c \frac{W}{m^{2}} & P_{F V}=P s r * \frac{R_{s o l}}{G_{s t d}}
\end{array}
$$

donde:

$\mathrm{P}_{\mathrm{Fv}}$ : potencia del generador $\mathrm{Fv}$

$\mathrm{G}_{\text {std }}$ : irradiancia solar en condiciones estandar

\subsection{Perfiles diarios de viento y de radiación}

Para el estudio planteado se requiere conocer la potencia disponible en los generadores durante las 24 horas del día, lo cual implica crear perfiles de viento y de radiación solar hora a hora. El perfil de velocidad del viento se obtiene multiplicando el valor estocástico de la velocidad media por un perfil de viento normalizado, que incluye una desviación. En la figura 1 los puntos rojos representan el perfil sin dispersión y los demás representan los perfiles cuando se incluye la dispersión.

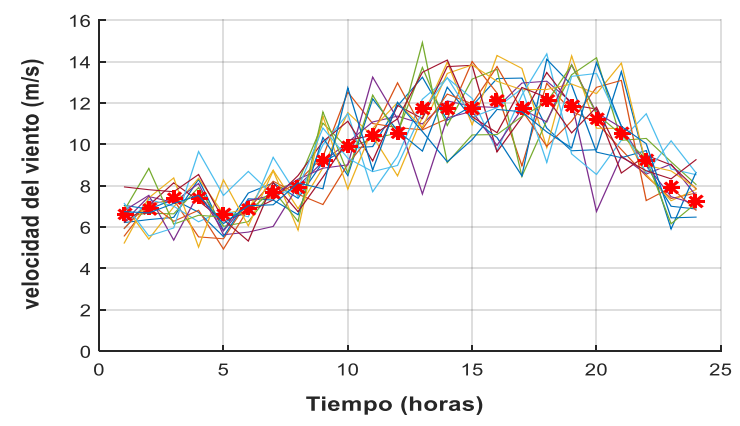

Figura 1. Representación estocástica del perfil de velocidades del viento. Fuente. Elaboración propia. 
El perfil de radiación se observa en la figura 2. Este perfil se obtiene multiplicando el valor estocástico de la radiación obtenida, por un perfil de radiación normalizado al cual se aplica una desviación hora a hora. La figura 2 evidencia un comportamiento estocástico que representa no solo los cambios en magnitud, sino también los cambios en la radiación producidos por condiciones de nubosidad.

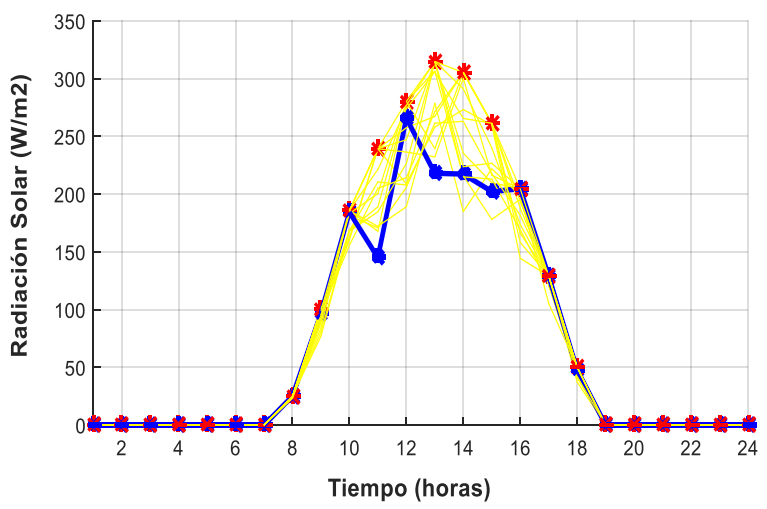

Figura 2. Representación estocástica del perfil de radiación solar diaria. Fuente. Elaboración propia.

\section{DESCRIPCIÓN DEL CASO DE ESTUDIO}

El caso de estudio plantea el despacho de energía y el cálculo del precio Spot del mercado en un sistema ideal compuesto por 4 plantas de generación, que deben suplir la demanda de potencia horaria representada por la curva de demanda. En cada caso se calcula la potencia disponible en las plantas intermitentes y se realiza el despacho usando la técnica de optimización de "Replicator dynamics", que es una técnica basada en la teoría de juegos y usa la utilidad marginal de las plantas de generación como criterio de optimización [18]. En este caso, el óptimo se alcanza cuando la utilidad marginal es igual para todas las plantas que despachan.

\subsection{Demanda de potencia}

Se representa mediante una curva de demanda horaria típica, con una demanda máxima de $515 \mathrm{MW}$ en la hora 20 (figura 3 ).

\subsection{Generación firme}

Se obtiene mediante una planta a gas de $198 \mathrm{MW}$, una de $160 \mathrm{MW}$ y una planta diesel de $265 \mathrm{MW}$, que pueden generar de manera continua durante las 24 horas del día. En este caso de estudio solamente se incluyen restricciones de capacidad de generación máxima, para las plantas en firme.

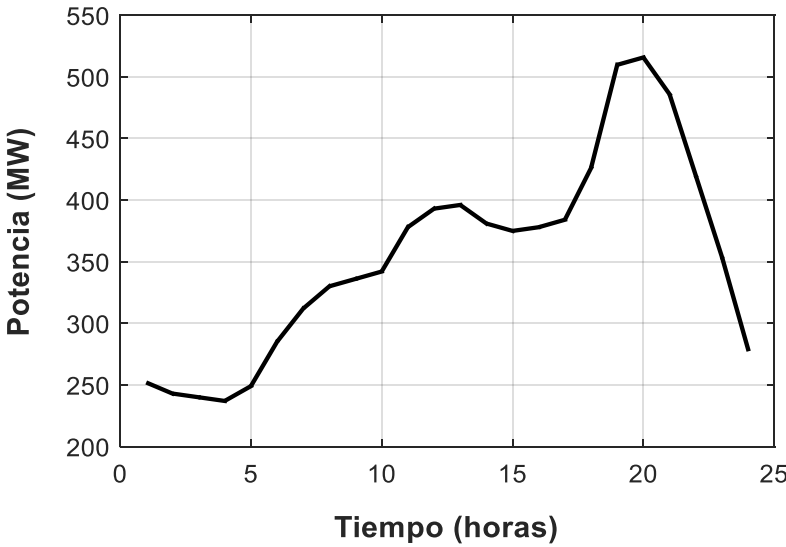

Figura 3. Curva de demanda de potencia. Fuente. Elaboración propia.

\subsubsection{Potencia Fotovoltaica.}

En este caso se tiene una representación estocástica que indica la potencia generada hora a hora y depende de los perfiles de radiación solar generados (Figura 4).

La potencia generada sigue la misma forma que la curva de radiación incidente en el panel.

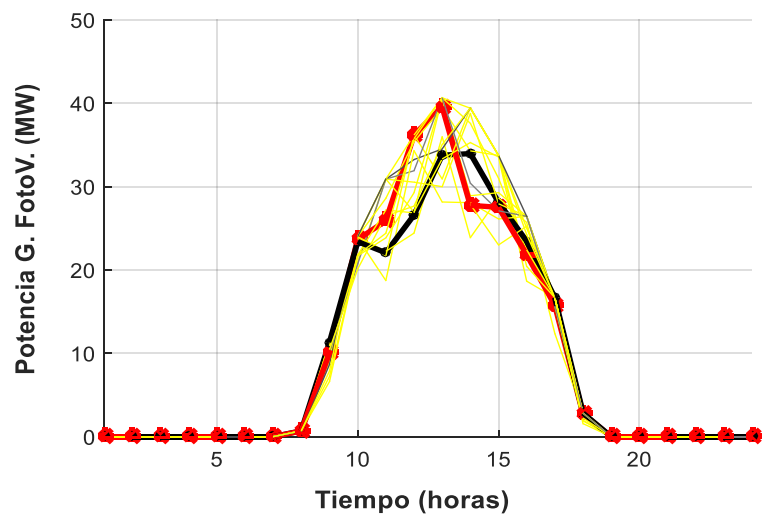

Figura 4. Potencia obtenida por el sistema fotovoltaico con representación estocástica. Fuente. Elaboración propia.

\subsubsection{Potencia eólica.}

Se calcula con los criterios indicados en el numeral 2.2. La figura 5 muestra varios perfiles entre los que se identifica uno en azul, que refleja las diferencias generadas por la representación estocástica. 


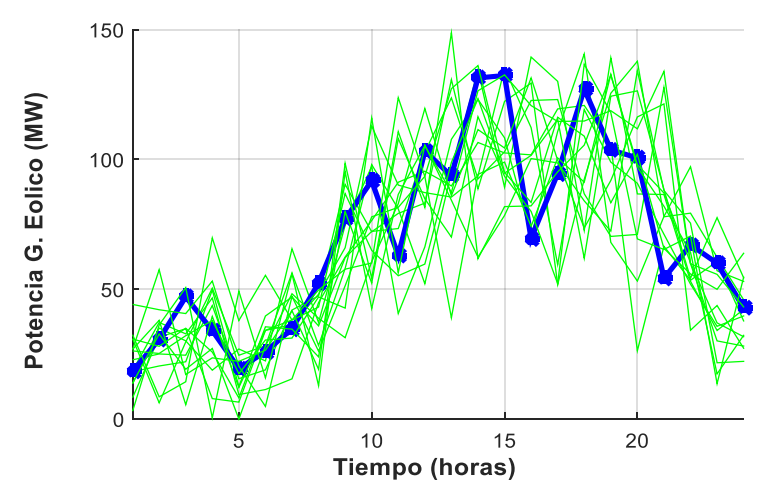

Figura 5. Potencia obtenida por la turbina eólica con representación estocástica. Fuente. Elaboración propia.

En este caso se pueden presentar caidas repentinas a cero cuando la velocidad del viento sobrepasa la velocidad de desconexión o cuando la velocidad es menor a la velocidad de arranque de la turbina.

\section{PLANTEAMIENTO DEL PROBLEMA}

El despacho ideal determina la cantidad de potencia a despachar por cada planta de generación (en firme e intermitentes), teniendo en cuenta sus límites máximos de generación hora a hora, con el fin de minimizar el costo del despacho. La técnica de replicator dynamics calcula el óptimo del sistema, maximizando la utilidad marginal de cada una de las unidades que despachan en el sistema. Esta condición de optimalidad se logra cuando las utilidades marginales de todas las plantas se hacen iguales.

El problema se expresa como indican las ecuaciones 6, 7 y 8 , presentadas a continuación

Max:

$$
J_{p}\left(p_{r}\right)=\sum_{i=1}^{N} J_{p i}\left(p_{r i}\right)
$$

Sujeto a:

Restricción de balance de potencia

$P_{L h}=\sum_{i=1}^{N} p_{r i}=\sum_{i=1}^{N} P_{G i h}$

Restricción de límite de potencia para cada planta $0 \leq p_{\text {ri }} \leq P_{\text {maxih }} \quad \forall i=1,2,3, \ldots \ldots N$

Donde :

$J_{p i}$ : función de utilidad marginal de la planta i $p_{r i}$ : potencia despachada por la planta $\mathrm{i}$

$P_{L h}$ : potencia total demandada en la hora $\mathrm{h}$

$P_{G i h}$ : potencia entregada por la planta i en la hora $\mathrm{h}$

$P_{\text {maxih }}$ : disponibilidad de potencia máxima para la planta i en la hora $h$.

Para el caso de las GEo y GFv, el límite de potencia $P_{\text {maxih }}$ cambia hora a hora, y dependen del valor calculado a partir de las funciones estocásticas, mientras que las plantas en firme se mantiene siempre constante.

La utilidad marginal de cada planta esta determinada por la función:

$J_{p i}\left(p_{r i}\right)=\frac{1}{c_{p i}}\left(2 p_{r i}-\frac{p_{r i}{ }^{2}}{P_{\max }}\right)$

Los cálculos se realizan mediante un proceso iterativo realizado en MATLAB®, usando un algoritmo en el que se han implementado las ecuaciones para el cálculo de los valores estocásticos de potencia en las plantas, así como las ecuaciones que describen el problema de estudio, incluyendo la función objetivo y las restricciones planteadas.

\section{SIMULACIONES Y RESULTADOS}

Para evaluar el impacto que tienen la radiación y la velocidad del viento en el despacho se analizan tres escenarios diferentes: en el primero se analiza el impacto de los sistemas GFv en el despacho sin tener en cuenta la GEo. En el segundo se analiza el efecto de la GEo sin incluir GFv, y en el tercer escenario se analiza la inclusión de los dos tipos de generación simultáneamente. En cada escenario se evalua: la potencia generada por el GFv y/o el GEo, su efecto en la utilidad marginal (fitness del algoritmo) y su efecto en la potencia despachada por cada una de las plantas.

\subsection{Escenario 1}

En este escenario se analizan dos casos de estudio: el primero con una potencia instalada del $10 \%$ en GFv respecto de la demanda máxima de $516 \mathrm{MW}$ (en la hora $20)$, y el segundo con una potencia instalada de $30 \%$ enGFv respecto de la demanda máxima.

Para el análisis estocástico se realizaron 100 simulaciones variando la radiación solar, y se calcularon la utilidad marginal y la potencia despachada por las plantas en cada caso.

En el primer caso (10\% de GFv) se evidencia un cambio en la utilidad marginal durante las horas en las que hay radiación solar (figura 6), afectando la cantidad de 
potencia despachada por las plantas en firme, tal como se ve en la figura 7.

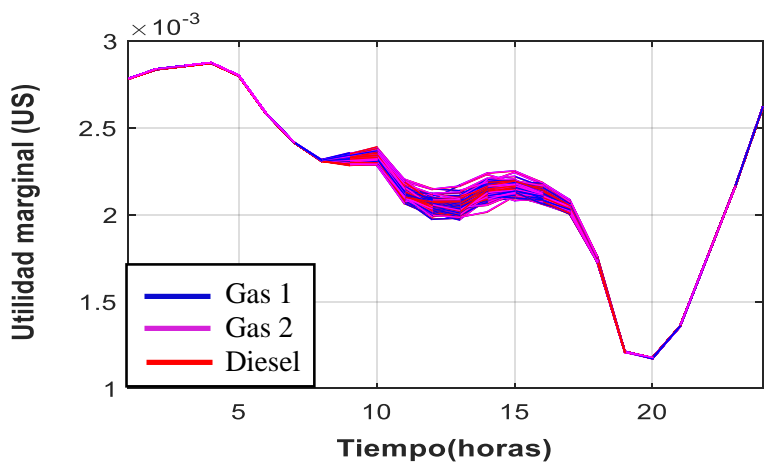

Figura 6. Utilidad marginal cuando se tiene $10 \%$ de GFv instalada. Fuente. Elaboración propia.

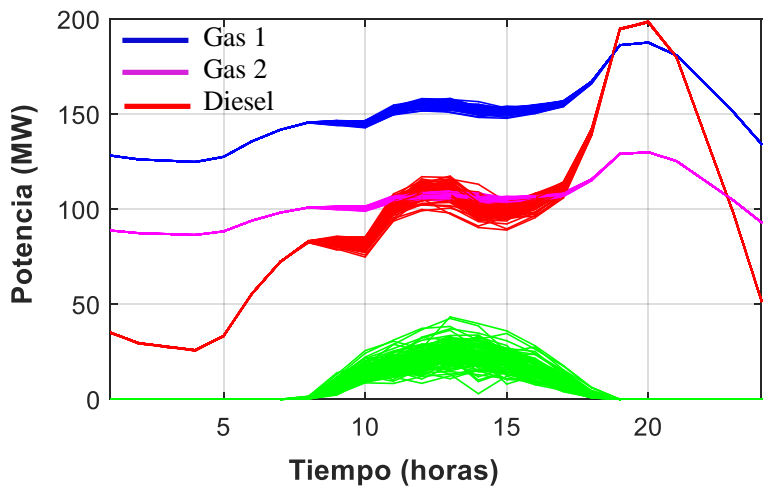

Figura 7. Potencia despachada por cada una de las plantas cuando se tiene $10 \%$ de GFv instalada. Fuente. Elaboración propia.

En la figura 7 se observa que la potencia despachada por las plantas en firme es alta cuando hay poca radiación, y disminuye cuando la radiación aumenta. Esto produce un incremento en la utilidad marginal con la que se asigna el despacho, porque la función de utilidad de las plantas tiene una relación inversa con la potencia despachada. En consecuencia, la GFv produce incrementos hasta de un $10 \%$ en la utilidad marginal, que pueden afectar el precio del mercado Spot.

Cuando la GFv instalada alcanza un 30\% de la demanda máxima se encuentran incrementos hasta del $21.9 \%$ en la utilidad marginal (figura 8). Aunque el método usado no calcula el precio de bolsa ni el precio al usuario final, estos podrían aumentar a causa de los incrementos en la utilidad marginal, para el caso de estudio. Es importante aclarar que contrario a este caso, en sistemas de potencia, la integración de las plantas renovables puede desplazar algunas plantas de generación firme durante el despacho, y esto disminuye el precio de bolsa.

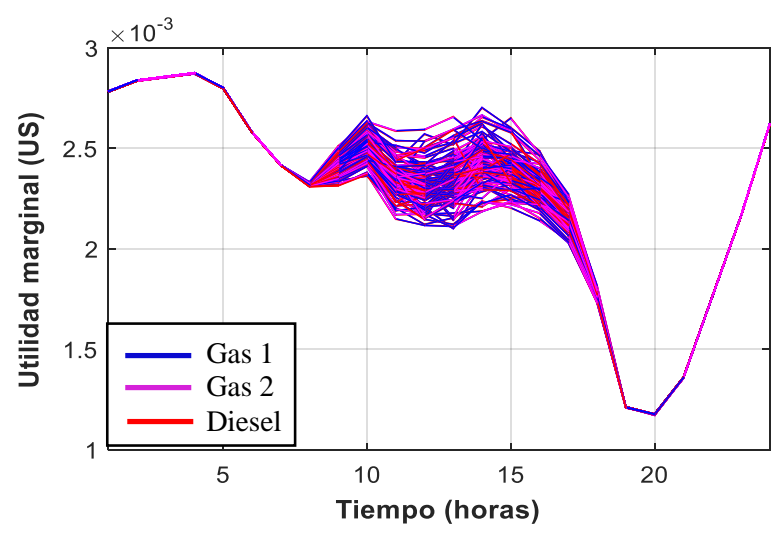

Figura 8. Utilidad marginal cuando se tiene $30 \%$ de GFv instalada. Fuente. Elaboración propia.

La potencia despachada por las plantas en firme se ve afectada por la inclusión de la GFv, dependiendo del costo de generación de cada planta. Las más afectadas serán aquellas con mayor sensibilidad a la variación de precio tal como sucede con las plantas térmicas a gas y diesel. En este caso (figura 9 ) la planta más afectada es la planta diesel (señal roja), que podría llegar a reducir la potencia hasta en un $58.6 \%$ según las simulaciones.

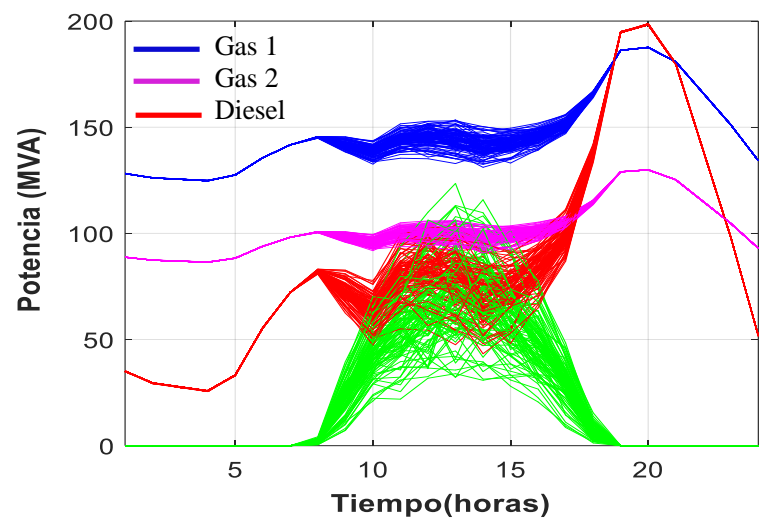

Figura 9. Potencia despachada por cada planta cuando se tiene $30 \%$ de GFv instalada. Fuente. Elaboración propia.

\subsection{Escenario 2}

En este escenario se analiza lo que sucede cuando se tiene un GEo con capacidad para entregar una potencia de hasta el $10 \%$ de la demanda pico.

Los resultados muestran cambios en la utilidad marginal durante las 24 horas del día porque el GEo funciona de manera continua, afectanto negativamente la potencia generada por las plantas en firme en todo momento (figura 10). 
A pesar de que la capacidad instalada es tan solo un $10 \%$ de la demanda, ésta puede tener un mayor impacto en el despacho de las plantas en firme comparada con el GFv, porque su efecto en el mercado estará presente durante todo el día.

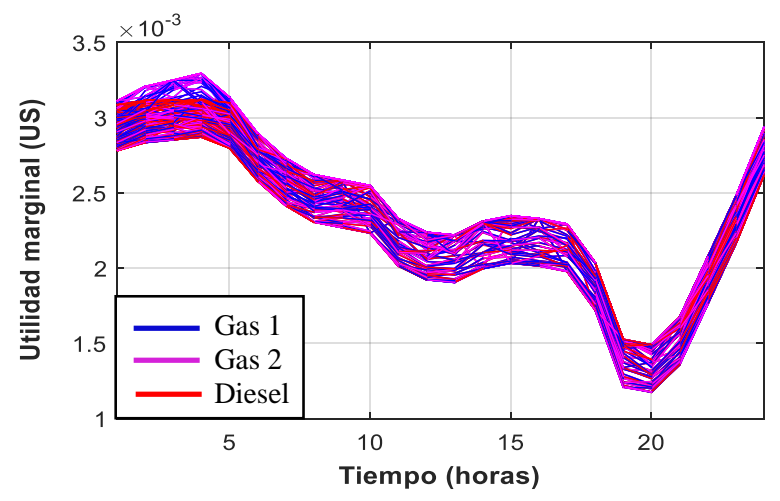

Figura 10. Utilidad marginal cuando se tiene $10 \%$ de GEo instalada. Fuente. Elaboración propia.

Un aspecto adicional observado en este caso es que la GEo puede afectar la potencia despachada por las plantas en firme hasta el punto de reducirlas a cero en momentos de baja demanda, como se ve en la figura 11. Esto indica que bajo ciertas condiciones, la planta diesel puede ser desplazada completamente por la GEo, como sucede en las primeras cinco horas del día.

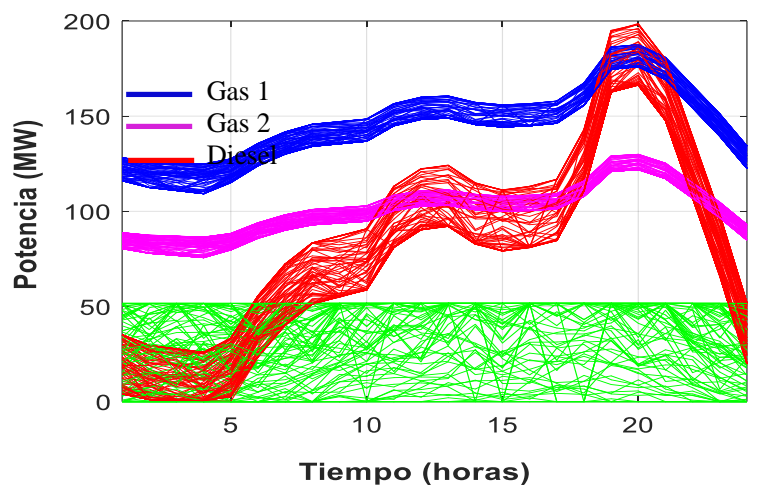

Figura 11. Potencia despachada por cada una de las plantas cuando se tiene $10 \%$ de GEo instalada. Fuente. Elaboración propia.

\subsection{Escenario 3}

En este escenario se tienen GEo y GFv operando de manera simultánea en el sistema, cada una con una capacidad instalada equivalente al $20 \%$ de la demanda pico.

La utilidad marginal (figura 12) refleja una variación similar a la observada en el escenario 2 , sin embargo esta diferencia se acentua en las horas que hay mayor participación del GFv.

La consecuencia de incluir estas dos plantas en el mercado es que, podría incrementar el precio de bolsa a causa del aumento en el ingreso marginal y que este se puede acentuar en horas con altos niveles de radición solar.

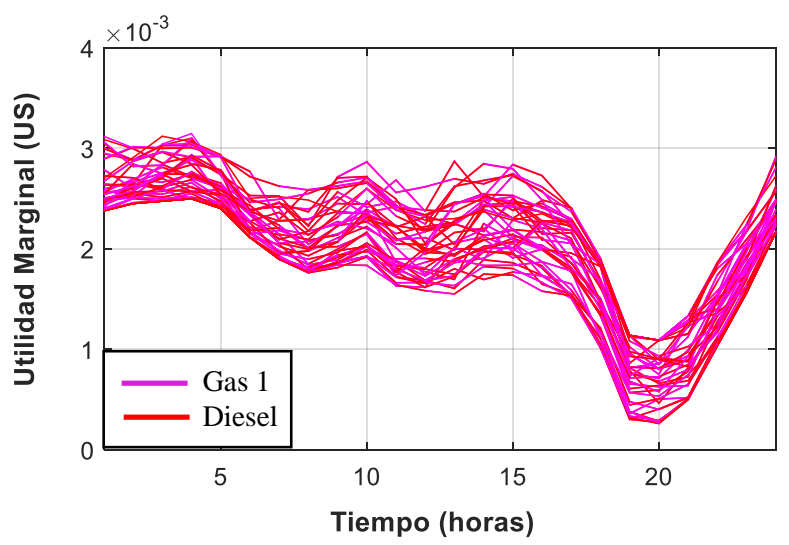

Figura 12. Utilidad marginal cuando se tiene $20 \%$ de GEo y $20 \%$ de GFv instalada. Fuente. Elaboración propia.

Esto indica que cuando la GEo y la GFv reciben beneficios despachando con un precio de oferta igual a cero (mercado no liberalizado), se podrían producir efectos negativos para los generadores. Frente a estos aspectos se hace necesario evaluar cuales son las condiciones adecuadas para hacer el despacho económico cuando se tienen sistemas de GEo y GFv, ya que el tipo de beneficios planteados en este caso de estudio reflejan una posible afectación para los competidores, para el mercado y para el usuario final, quien debe asumir los costos asociados a este tipo de estrategias.

\section{CONCLUSIONES}

La inclusión de las plantas GEo y GFv en el despacho tienen un impacto negativo en todas las plantas de generación firme conectadas en la microrred, porque obligan a reducir la potencia despachada por cada una de estas. Ésta reducción tienen mayor efecto en las plantas con tecnología diesel, para el caso de estudio.

La estrategia de despacho con precio de oferta cero para las plantas GFv y GEo obliga a reducir la potencia generada por las plantas en firme hasta en un $58.6 \%$ en situaciones donde se tiene alta demanda de potencia y elevada participación de GFv y GEo. Además, puede reducir a cero la potencia despachada por las plantas en 
firme cuando se tienen una baja demanda de potencia y una elevada generación eólica, situación que se pueden observa en horas nocturnas.

La GEo tienen mayor impacto negativo sobre las plantas en firme porque hay menor dispersion en el recurso eólico y puede suministrar potencia durante las 24 horas del día. Esta situación hace que las plantas en firme dejen de suministrar una mayor cantidad de energía-día cuando se tienen GEo, a la dejada de suministrar cuando se tienen solamente GFv, lo cual disminuye sus ingresos y rentabilidad.

\section{AGRADECIMIENTOS}

Los autores dan agradecimientos a la Universidad Nacional de Colombia y a la Universidad Distrital Francisco José de Caldas por el apoyo para el trabajo de investigación.

\section{REFERENCIAS}

[1] A. S. Brouwer, M. van den Broek, A. Seebregts, and A. Faaij, "Impacts of large-scale Intermittent Renewable Energy Sources on electricity systems, and how these can be modeled," Renew. Sustain. Energy Rev., vol. 33, pp. 443-466, May 2014.

[2] C. B. B.M. Buchholz, "The impact of dispersed power generation in distribution systems," Qual. Secur. Electr. Power Deliv. Syst. 2003. CIGRE/IEEE PES Int. Symp., pp. 198-203, 2003.

[3] G. Kosmadakis, S. Karellas, and E. Kakaras, "Renewable and Conventional Electricity Generation Systems: Technologies and Diversity of Energy Systems," Renew. Energy Gov., vol. 23, pp. 9-30, 2013.

[4] K. Würzburg, X. Labandeira, and P. Linares, "Renewable generation and electricity prices: Taking stock and new evidence for Germany and Austria," Energy Econ., vol. 40, pp. 159-171, 2013.

[5] L. Dusonchet and E. Telaretti, "Comparative economic analysis of support policies for solar PV in the most representative EU countries," Renew. Sustain. Energy Rev., vol. 42, pp. 986-998, 2015.

[6] L. Gabriel and M. Díaz, "Comparación de métodos de asignación a redes para distintos volumenes de transito," Rev. UIS Ing., vol. 9, no. 1, pp. 77-84, 2010.

[7] V. Toro-tovar, S. Rivera, and E. Mojica-nava, "Mejoras de la regulación de frecuencia utilizando el aumento de inercia de microrredes interconectadas," Rev.
UIS Ing., vol. 16, no. 2, pp. 35-42, 2017.

[8] O. González, A. Pavas, and S. Sánchez, "Cuantificación del ahorro de energía eléctrica en clientes residenciales mediante acciones de gestión de demanda," Rev. UIS Ing., vol. 16, no. 2, pp. 217-225, 2017.

[9] R. Wilson, "Architecture of Power Markets," Econométrica, vol. 70, no. 4, pp. 1299-1340, 2002.

[10]A. H. Fathima and K. Palanisamy, "Optimization in microgrids with hybrid energy systems - A review," Renew. Sustain. Energy Rev., vol. 45, pp. 431-446, May, 2015.

[11]A. Brooks, E. Lu, D. Reicher, C. Spirakis, and B. Weihl, "Demand dispatch," IEEE Power Energy Mag., vol. 8, no. 3, pp. 20-29, 2010.

[12]N. C. Abdullah Urkmez, "Determining Spot price and economic dispatch in deregulated power systems," $J$. Math. Comput. Appl., vol. 15, no. 1, pp. 25-33, 2010.

[13]A. Altunkaynak, T. Erdik, and S. Zekai, "Theoretical derivation of wind power probability distribution function and applications," J. Appl. Energy, vol. 92, pp. 809-814, 2012.

[14]S. Mohammadi, S. Soleymani, and B. Mozafari, "Scenario-based stochastic operation management of MicroGrid including Wind, Photovoltaic, MicroTurbine, Fuel Cell and Energy Storage Devices," Int. J. Electr. Power Energy Syst., vol. 54, pp. 525-535, Jan. 2014.

[15]D. Arango and R. Urrego, "Despacho económico en microredes con penetración de energía renovable usando algoritmo de punto interior y restricciones lineales," Ing. y Cienc., vol. 13, no. 25, pp. 123-152, 2017.

[16]J. Arevalo, F. Santos, and S. Rivera, "Application of analytical uncertainty costs of solar, wind and electric vehicles in optimal power dispatch," Rev. Ing., vol. 22, no. 3, pp. 1-23, 2017.

[17]S. Mathew, G. S. Philip, and C. M. Lim, "Analysis of Wind Regimes and Performance of Wind Turbines," Adv. Wind Energy Convers. Technol., pp. 71-83, 2011.

[18]E. Mojica-Nava, C. Barreto, and N. Quijano, "Population Games Methods for Distributed Control of Microgrids," IEEE Trans. Smart Grid, vol. PP, no. 99, pp. 1-1, 2015. 